\title{
Role of Cys73 in the thermostability of farnesyl diphosphate synthase from Geobacillus stearothermophilus
}

\author{
Petrus Yesaya Samori ${ }^{1} \cdot$ Koki Makabe $^{1} \cdot$ Norimasa Ohya $^{2} \cdot$ Bunpei Hatano $^{1}$. \\ Satoshi Murakami ${ }^{1} \cdot$ Tatsuro Kijima $^{1}{ }^{1}$
}

Received: 13 May 2017/ Accepted: 14 June 2017/Published online: 10 July 2017

(c) The Author(s) 2017. This article is an open access publication

\begin{abstract}
Farnesyl diphosphate synthase (FPPase) is an enzyme that catalyzes the condensation between one molecule of dimethylallyl diphosphate (DMAPP) and two molecules of isopentenyl diphosphate (IPP) to produce farnesyl diphosphate (FPP). FPP is an important precursor in the isoprenoid synthesis pathway. In this study, the crystal structure of FPPase from Geobacillus stearothermophilus (GsFPPase) was determined at $2.31 \AA$ resolution. The structure of GsFPPase shows a three-layered all $\alpha$ helical fold and conserved functional domains similar to other prenyltransferases. We have analyzed the structural features of GsFPPase related to thermostability and compared it with those of human and avian mesophilic FPPases. "Semi-conserved" regions which appear to be possible features contributing to the thermostability of FPPase were found.
\end{abstract}

Keywords Farnesyl diphosphate synthase - Geobacillus stearothermophilus · Thermostability · Crystal structure · Thermal unfolding simulation

Tatsuro Kijima

kijima@yz.yamagata-u.ac.jp

1 Department of Biochemical Engineering, Graduate School of Science and Engineering, Yamagata University, 4-3-16 Jonan, Yonezawa, Yamagata 992-8510, Japan

2 Department of Material and Biological Chemistry, Faculty of Science, Yamagata University, 1-4-12 Kojirakawa, Yamagata, Yamagata 990-8560, Japan

\section{Introduction}

Isoprenoids are a highly diverse group of compounds which play important roles in the physiological processes of all free-living organisms. The fundamental step in the biosynthesis of isoprenoids is the chain elongation of prenyl diphosphate precursors by prenyltransferases. These enzymes catalyze the successive condensation between isopentenyl diphosphate (IPP, C5) and allylic diphosphates to produce prenyl diphosphates with various chain lengths and stereochemistries, thus, they are commonly classified based on the chain length and the geometry of the double bond of the final product that is formed by the reaction (Ogura and Koyama 1998).

Farnesyl diphosphate synthase (FPPase; EC 2.5.1.10) is one of the prenyltransferases and occupies a central branch point in the prenyl chain elongation pathway. It catalyzes the condensations of IPP with dimethylallyl diphosphate (DMAPP, C5) and with geranyl diphosphate (GPP, C10) to give the key precursor $(2 E, 6 E)$-farnesyl diphosphate (FPP, C15) as the final product (Eberhardt and Rilling 1975).

Because of its critical role in the pathway, FPPase has been used in both mechanistic and synthetic studies. Poulter and Rilling used FPPase from avian liver and porcine liver to study the mechanism of chain elongation (Poulter and Rilling 1978). Koyama et al. used porcine liver FPPase to develop a novel chiral synthesis method and successfully applied the method to the chiral synthesis of faranal and 4-methyl-juvenile hormone (Koyama et al. 1980; Kobayashi et al. 1980; Koyama et al. 1987). Also, Maki et al. showed that porcine liver FPPase can be used to synthesize the butterfly hair-pencil pheromone (Maki et al. 1995). These studies have demonstrated that FPPase has potential applications in the synthesis of a great variety of organic compounds. However, the practical uses of FPPase 
in industrial applications are limited due to the instability of the enzyme and the difficulty in obtaining it in significant amounts.

Thermostable FPPase is becoming an increasing necessity to provide an enzyme which is more stable and more compatible with industrial application. Genes encoding thermostable FPPases have been isolated and prepared from some thermophilic bacteria. For example, bifunctional enzyme farnesyl diphosphate/geranylgeranyl diphosphate synthase and the gene encoding it were isolated from high thermophile Methanobacterium thermoauototrophicum, and the enzyme showed a high thermal stability (Chen and Poulter 1993). A gene encoding thermophilic FPPase was also isolated from medium thermophile Geobacillus stearothermophilus (Bacillus stearothermophilus) and was efficiently overexpressed in Escherichia coli host cells (Koyama et al. 1993).

Geobacillus stearothermophilus FPP synthase (GsFPPase) has been shown to display thermal stability properties. The enzyme is not deactivated even after heat treatment at $65^{\circ} \mathrm{C}$ for $100 \mathrm{~min}$. Although many years have passed since studies of GsFPPase began, the factors that determine its thermostable properties still remain unclear.

In their studies on the roles of cysteine residues in GsFPPase, Koyama et al. found that replacing Cys residue at position 73 with Phe caused the enzyme to become very sensitive to heat treatment (Koyama et al. 1994). Here, we verified the effect of Cys73 on the thermostability of GsFPPase by solving its crystal structure at a resolution of $2.31 \AA$ and conducted thermal unfolding simulations.

\section{Materials and methods}

\section{Expression and purification}

Wild-type GsFPPase was overexpressed and purified according to the method reported by Koyama et al. (1993), with some modifications. E. coli cells harboring expression plasmid that carry wild-type GsFPPase gene were cultured overnight in Luria Broth (LB) medium containing $100 \mu \mathrm{g} /$ $\mathrm{ml}$ of ampicillin at $37{ }^{\circ} \mathrm{C}$. Cultured cells were transferred to 100 volumes of the same fresh medium and were grown further at $37{ }^{\circ} \mathrm{C}$ to an approximate $\mathrm{OD}_{600}$ value of 0.6. Isopropyl $\beta$-D-1- thiogalactopyranoside (IPTG) was then added to a final concentration of $0.1 \mathrm{mM}$ and the solution was incubated overnight. The cells were harvested and disrupted by sonication in $10 \mathrm{mM}$ Tris- $\mathrm{HCl}$ buffer $(\mathrm{pH}$ 8.0). The homogenate was heated at $55^{\circ} \mathrm{C}$ for $60 \mathrm{~min}$, fractionated with $35-60 \%$ ammonium sulfate solution, and purified using an anion exchange chromatography column DEAE-TOYOPEARL (Tosoh Corporation, Japan).

\section{Crystallization and data collection}

The purified protein sample was dialyzed against $10 \mathrm{mM}$ Tris-HCl buffer (pH 7.0) and concentrated to $14.5 \mathrm{mg} / \mathrm{mL}$ using Amicon Ultra concentrator (MWCO 10,000 Da; Millipore, USA). Initial crystal screening was performed by the sitting-drop vapor diffusion method using Crystal Screens I and II (Hampton Research, USA) and JCSG+ (Qiagen, USA) sparse matrix screening kit. Optimization was performed using the hanging drop vapor-diffusion method, where a drop initially contained $1 \mu \mathrm{L}$ each of the reservoir solution and purified protein sample. Crystals for data collection were obtained using a reservoir solution consisting of $0.1 \mathrm{M}$ Tris- $\mathrm{HCl}$ (pH 8.5), 34\% PEG400, and $0.1 \mathrm{M} \mathrm{LiCl}$ at $37{ }^{\circ} \mathrm{C}$.

\section{Structure determination}

Crystals of GsFPPase were frozen in liquid nitrogen using the crystallization solution as a cryoprotectant (34\% PEG400). The X-ray diffraction data were collected at the BL-5A beamline (KEK Photon Factory, Tsukuba, Japan). The diffraction images were processed with HKL2000 (Otwinowski and Minor 1997) and SCALA of the CCP4 program suite (CCP4 1994). The structure of GsFPPase was determined by molecular replacement using the program MOLREP in CCP4 using FPP synthase structure from Staphylococcus aureus (PDB ID 1RTR) as a search model. In the further refinement, 5\% randomly chosen reflections were set aside for calculating R-free. Refinement was carried out with Refmac5 in CCP4. The model building was carried out using the Coot program. The coordinates have been deposited in the PDB with entry code 5AYP. Molecular graphics were generated using 5AYP. The data collection and refinement statistics are summarized in Table 1.

\section{Melting point analysis}

The thermal stability of GsFPPase was measured using differential scanning calorimetry (DSC; VP-capillary DSC, GE healthcare, USA). A protein sample with $1 \mathrm{mg} / \mathrm{mL}$ in a $50 \mathrm{mM}$ phosphate (pH 7.0) and $100 \mathrm{mM} \mathrm{NaCl}$ buffer was used. Scanning speed was $60{ }^{\circ} \mathrm{C} / 1 \mathrm{~h}$.

\section{Missing loops modeling, point mutation modeling, and thermal unfolding simulation}

Insertion of the missing loops of GsFPPase was carried out using the ab initio method implemented in Modeller 9.16 (Fiser et al. 2000). The quality of each model was validated using ProSa (Wiederstein and Sippl 2007) and SAVES server (Laskowski et al. 1996). The resulting structure with 
the best quality was then used to construct the point-mutated enzyme C73F structure using Modeller 9.16. The C73F model was minimized further with the steepest descent algorithm followed by the conjugate gradient algorithm by USFC Chimera (Pettersen et al. 2004), which was developed by the Resource for Biocomputing, Visualization, and Informatics at the University of California, San Francisco. Thermal unfolding simulations were performed using CNA web server (http://cpclab.uni-duesseldorf.de/cna/) (Krüger et al. 2013). CNA web server is a web interface for performing rigidity theory-based thermal unfolding simulations based on Constrain Network Analysis (CNA) approach (Pfleger et al. 2013a). CNA models a protein as a constraint network, and by continuously removing non-covalent constraints (i.e., hydrogen bonds) from the network, it simulates the thermal unfolding process of the protein.

In this study, the simulation performed by CNA was carried out with the 'ensemble of network-single structure' analysis type for each enzyme, number of network topologies in each ensemble was set to 50. The network ensembles are generated from a single input structure using fuzzy-constraint definitions implemented in CNA. For the simulation parameters, E-cutoff for hydrogen bonds was set to default and hydrophobic cutoff was treated in a temperature-dependent manner. More specifically, by decreasing the E-cutoff for hydrogen bonds from an initial value of -0.1 to $-6.0 \mathrm{kcal} \mathrm{mol}^{-1}$ with a step size of $0.1 \mathrm{kcal} \mathrm{mol}^{-1}$, hydrogen bonds were removed from the network in the bond strength order, based on the idea that stronger hydrogen bonds break at higher temperature. On the other hand, more hydrophobic tethers were added to the network by increasing the hydrophobic cutoff from an initial value of $0.25 \AA$ to a terminal value of $0.40 \AA$, based on the fact that strength of hydrophobic interactions increases with increasing temperature (Schellman 1997).

CNA performs rigidity analysis of a protein by computing global and local indices. Global indices are used to identify phase transition points $T_{\mathrm{p}}$, where the network shifts from rigid to flexible, while local indices are used to characterize the flexibility of each bond in the network. In the case of global indices, here we only consider the cluster configuration entropy $H_{\text {type2 }}$, because $T_{\mathrm{p}}$ which are calculated using this modified $\mathrm{H}$ have been found to be related to the thermostability of proteins, i.e., the melting temperatures $\left(T_{\mathrm{m}}\right)$ of proteins (Radestock and Gohlke 2008, 2011; Pfleger et al. 2013b).

\section{Results and discussion}

\section{Overall structure of GsFPPase}

The apo form of GsFPPase was crystallized in a $P 2{ }_{1} 2_{1} 2_{1}$ space group with one homodimer in the asymmetric unit. GsFPPase homodimer is stabilized by hydrophobic interactions involving helices $\alpha 5$ and $\alpha 6$ from both monomers. The dimer interface buries $3350 \AA^{2}$ of accessible surface area per monomer (Fig. 1a). Because GsFPPase is active as a homodimer (Koyama et al. 2000), it is reasonable to consider that the dimer occurs in solution has a similar
Fig. 1 Overall structure of GsFPPase. a Cartoon representation of $G s$ FPPase homodimer. Chain A and chain $\mathrm{B}$ are shown in light green and pink, respectively. Connecting loops are shown in orange. b Monomeric structure of GsFPPase, with $\alpha$-helices, $N$ terminal, and $C$-terminal are marked correspondingly. c Tube representation of GsFPPase monomer. Five conserved regions that form the wall of the central cavity (yellow mesh) and the first aspartate-rich motif (FARM) are shown in blue and red, respectively. Two semiconserved regions are shown in magenta. Cys73 (inset, lime green mesh represent $2 \mathrm{Fo}-\mathrm{Fc}$ electron density map contoured at $1.0 \sigma$ ) is located on the semiconserved region of helix $\alpha 4$ (a)

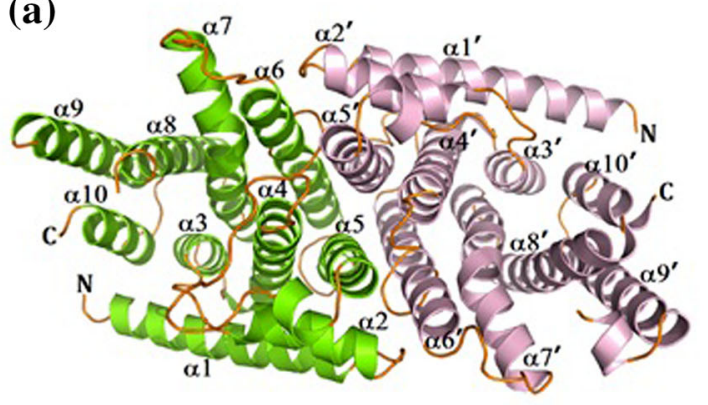

(b)

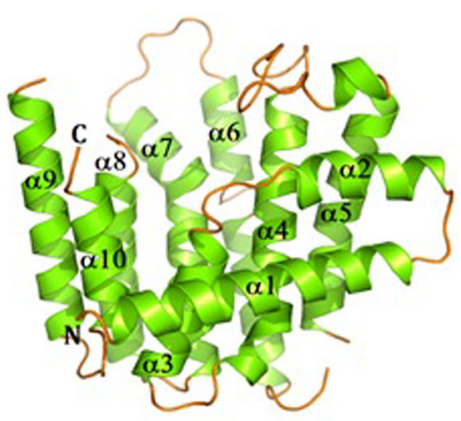

(c)

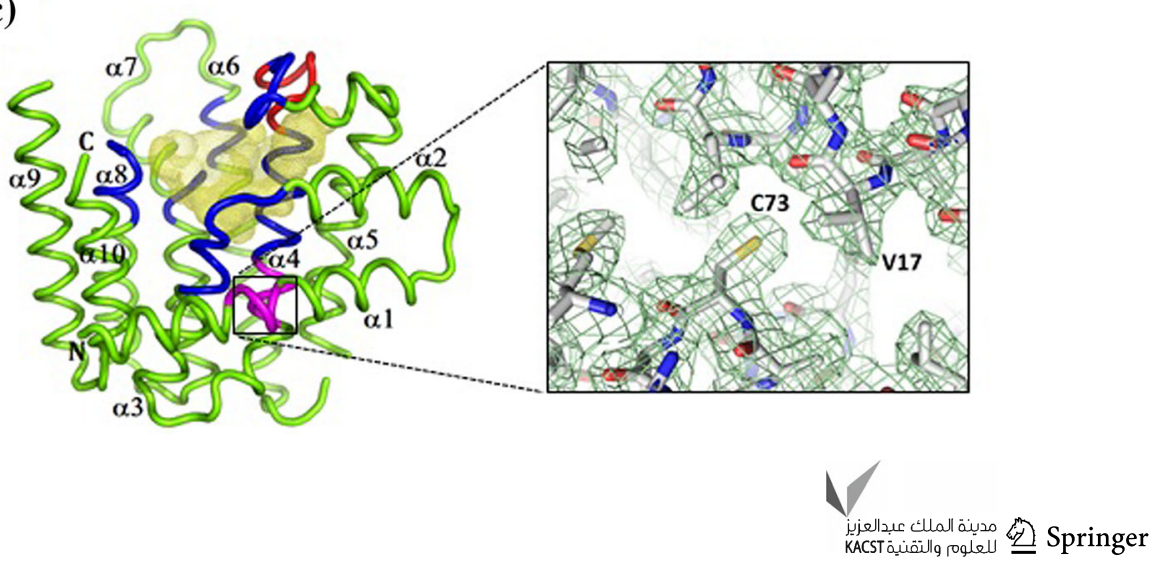


(a)

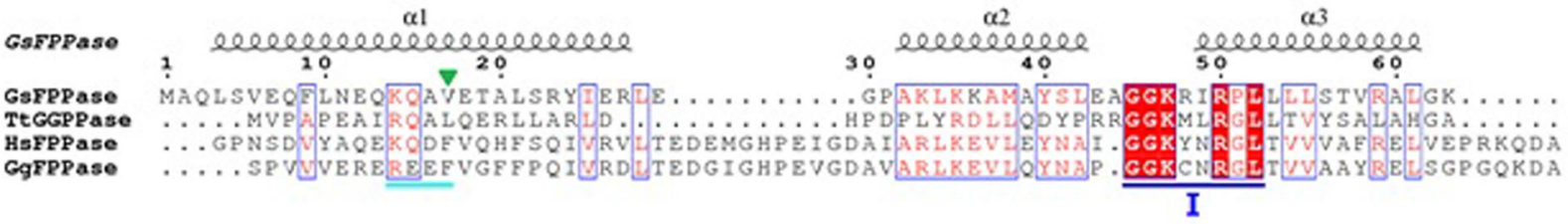

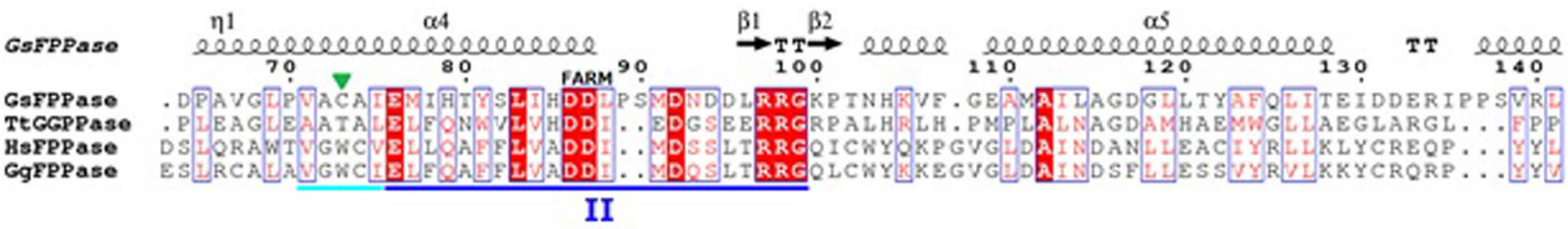

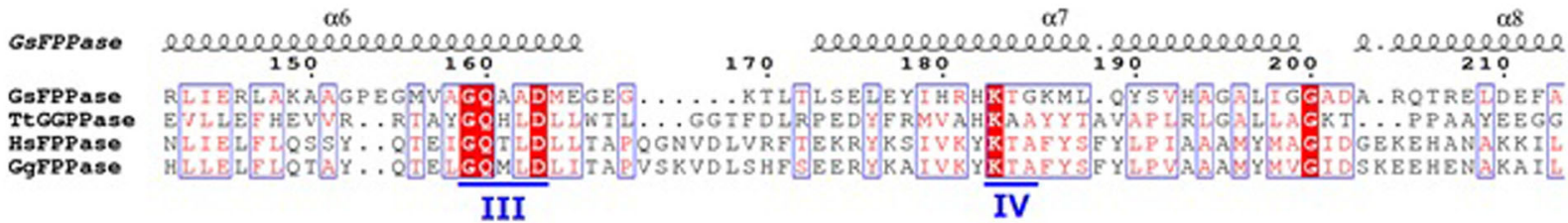
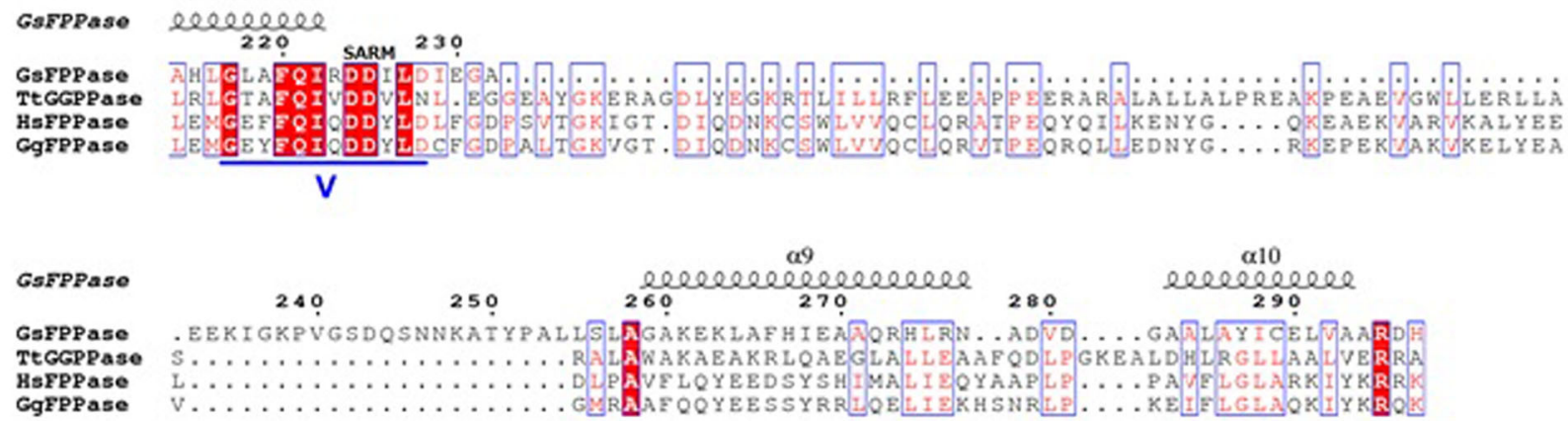

(b)

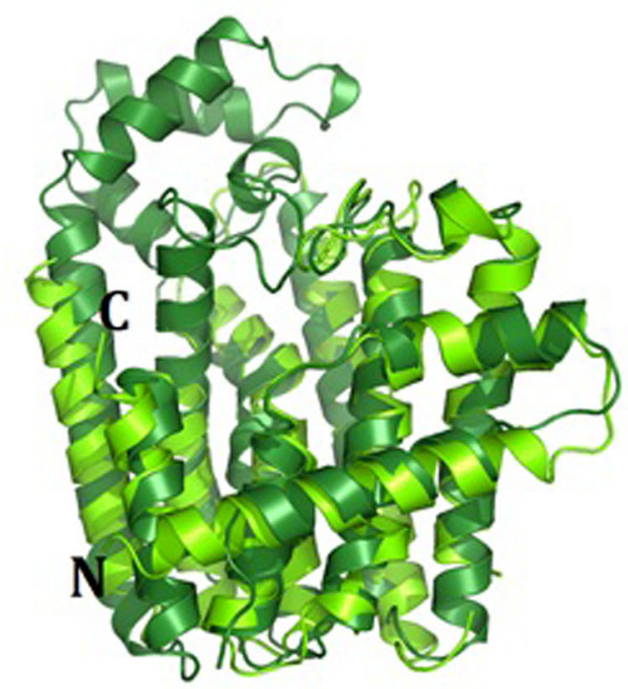

orientation with molecules in the crystal lattice shown in

Fig. 1a.

Like FPPases from other species, each monomer of GsFPPase exhibits a three-layered all $\alpha$-helical

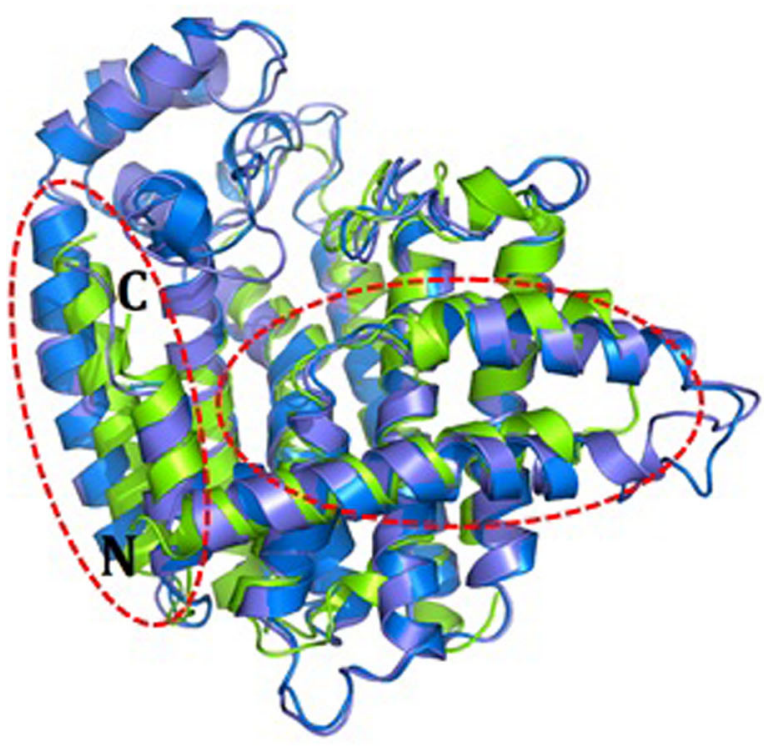

prenyltransferase fold. The typical fold consists of ten $\alpha$ helices and connecting loops, with the first, second, and third layer formed by helices $\alpha 1$ and $\alpha 2$, helices $\alpha 3-\alpha 5$ and $\alpha 10$, and helices $\alpha 6-\alpha 9$, respectively (Fig. 1b). A large 
४Fig. 2 Structural comparison of GsFPPase to its homologues from other species. a Multiple sequence alignment of GsFPPase with three selected prenyl diphosphate synthases. Five conserved regions, I-V, are underlined in blue. Semi-conserved regions are underlined in cyan. Two residues affecting atomic packing (e.g., Val17 and Cys 73 in the case of GsFPPase) are indicated by green inverted triangles. The alignment was created using ESPript (Robert and Gouet 2014). (GsFPPase, Geobacillus stearothermophilus FPP synthase (Uniprot ID: Q08291); TtGGPPase, Thermus thermophilus GGPP synthase (Q5SMD0); HsFPPase, Homo sapiens FPP synthase (P14324); GgFPPase, Gallus gallus FPP synthase (P08836)). b Superimposition between $G s$ FPPase and three selected prenyl diphosphate synthases. $G s$ FPPase, $T t$ GGPPase, human FPPase, and avian FPPase are colored in light green, dark green, blue, and light purple, respectively

central cavity located within the bundle of these $\alpha$-helices is the putative active site. The wall of this cavity contains five amino acid sequences that are highly conserved among prenyltransferases (Fig. 1c). Conserved first aspartate-rich motif [FARM, DDXX (XX) D: residues 86-92], was found in helix $\alpha 4$, located on the surface of the cavity. By contrast, second aspartate-rich motif (SARM, DDXXD: residues 224-228), which is supposed to exist in $\alpha 8$ on the opposite wall from FARM, could not be traced in the electron density map. This is because SARM is located near a flexible loop connecting $\alpha 8$ and $\alpha 9$ (residues 229-256), whose flexibility caused the electron density of SARM and the loop itself to become invisible in the apoGsFPPase structure. There is also a small missing loop between $\alpha 4$ and $\alpha 5$ containing residues 130 and 131. Cys 73 is a part of a "semi-conserved" region and is located in helix $\alpha 4$, exactly on the intersection point of orthogonally positioned $\alpha 1$ and $\alpha 4$ (Fig. 1c). Cys73 and the "semiconserved" region will be further discussed later.

\section{Structural comparison of GsFPPase with mesophilic FPPases}

Many studies have been conducted to elucidate the factor(s) that determine thermostability of an enzyme, and the often used method is based on comparison of thermophilic enzymes and their mesophilic homologs. Because prenyltransferases can be divided into prokaryotic and eukaryotic families (Chen et al. 1994), comparison of GsFPPase with both prokaryotic and eukaryotic family members might give some clues about the determinant(s) of its thermostability.

The structure of GsFPPase was compared with Thermus thermophilus geranylgeranyl diphosphate synthase (TtGGPPase), human FPPase, and avian FPPase. Human and avian FPPases were selected as representatives of mesophilic FPPases from the eukaryotic family. $T t$ GGPPase was chosen because it is the only thermophilic prenyltransferase from the prokaryotic family whose crystal structure has been solved and is relatively similar to FPPase structure.

Structure-based multiple sequence alignment of the four selected enzymes showed that these enzymes share five common conserved regions (I-V) of the prenyltransferase family. Two sequences which appear to be semi-conserved regions were also spotted in helices $\alpha 1$ and $\alpha 4$, with one of the regions located before region II (Figs. 1c, 2a). We called these regions "semi-conserved" because residues in these regions are similar between intra-family members but differ for inter-family members. For the eukaryotic families, the semi-conserved region before region II contains a GWC (Glycine-Tryptophan-Cysteine) motif. On the other
Fig. 3 Structural surroundings of semi-conserved regions. Two important residues which affect the atomic packing of helices $\alpha 1$ and $\alpha 4$ are shown in stick and sphere models (orange) and residues involved in the interactions are shown as stick models and labeled. Hydrogen bonds are indicated by dashed lines (black). a Semi-conserved regions of GsFPPase. b Semiconserved regions of $T t$ GGPPase. c Semi-conserved regions of human FPPase. d Semi-conserved regions of avian FPPase (a)

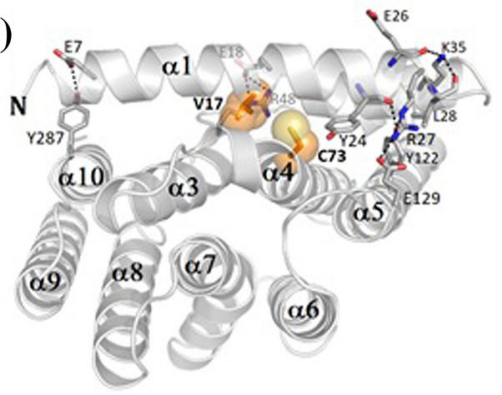

(c)

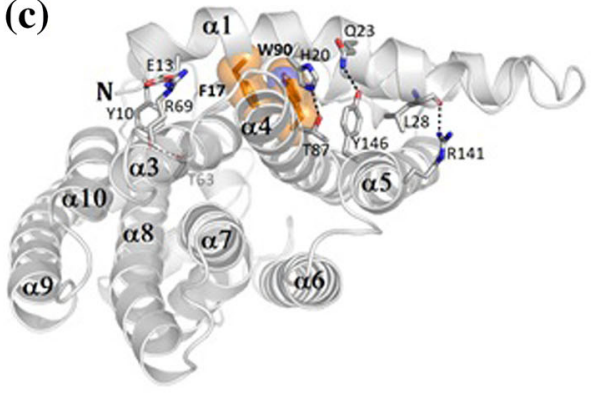

(b)

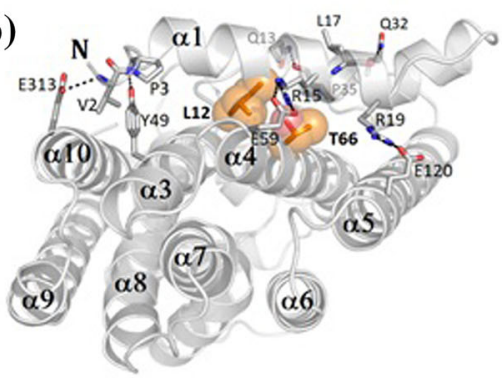

(d)

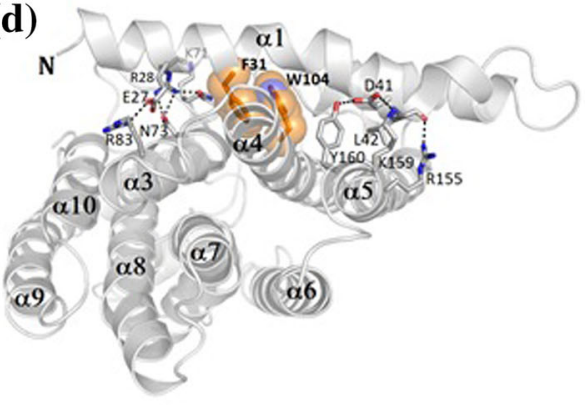

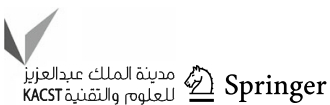


Fig. 4 Comparison of GsFPPase to $\mathrm{SaFPPase}$. a Sequence alignment of GsFPPase with $\mathrm{SaFPPase.}$ Conserved regions I and II are underlined in blue, and semiconserved regions are underlined in cyan. Two residues affecting atomic packing are indicated by green inverted triangles. b Structural surroundings of semi-conserved regions of GsFPPase (left) and SaFPPase (right). Two important residues which affect the atomic packing of helices $\alpha 1$ and $\alpha 4$ are shown by sphere models (orange) (a)
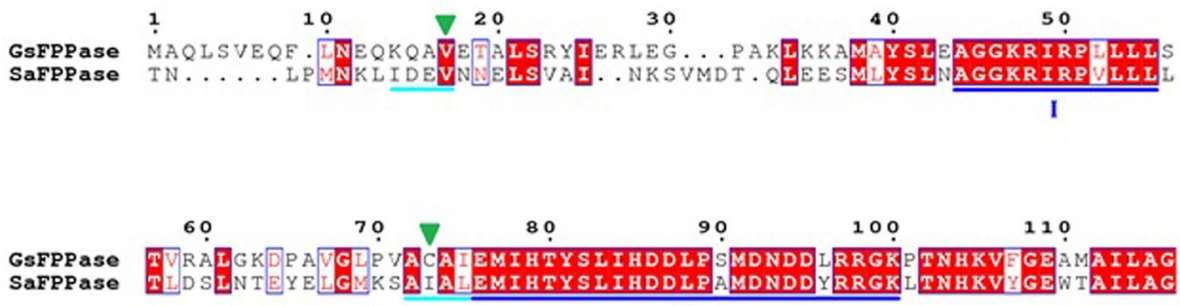

(b)

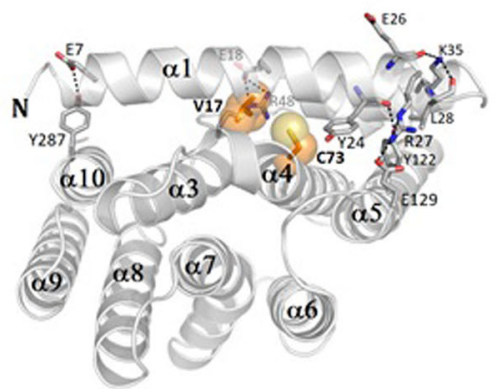

hand, the corresponding position in the eukaryotic families contains an AXA (Alanine-Unknown-Alanine) motif. Superimposition of the structure of GsFPPase with three other selected prenyltransferases indicated that the overall folding pattern of GsFPPase is highly similar to the other enzymes, especially to $T t$ GGPPase. However, there are significant differences between GsFPPase and its mesophilic homologs, in terms of the conformations of helices $\alpha 1, \alpha 2, \alpha 9$, and $\alpha 10$ (indicated with red-dashed lines in Fig. 2b). The differences may be attributed to the differences in semi-conserved regions. Differences in size of amino acids in these regions give rise to differences in the tightness of atomic packing of helices $\alpha 1$ and $\alpha 4$, which also affect the overall packing of the surrounding helices (Fig. 3). It seems that atomic packing of orthogonally positioned helices $\alpha 1$ and $\alpha 4$ is most likely influenced by two residues that are upside down relative to each other on the intersection point of $\alpha 1$ and $\alpha 4$. In the case of the eukaryotic family members, the two residues, which are Phe17 and Trp90 in human FPPase or Phe31 and Trp104 in avian FPPase, tend to be more bulky than in thermophilic homologs from the prokaryotic family. The pi-pi stacking interaction of these two residues cause the mesophilic enzymes to have a better or tighter atomic packing of helices $\alpha 1$ and $\alpha 4$, which then causes the atomic packing of other helices, especially between $\alpha 1$ and $\alpha 10$, to become looser (Fig. 3c, d). On other hand, the two residues that are located in the same position in GsFPPase and $T t$ GGPPase are relatively small, thus the atomic packing of $\alpha 1$ and $\alpha 4$ is relatively loose. However, the overall atomic packing of surrounding helices, e.g. atomic packing between $\alpha 1$ and $\alpha 10$, is tight (Fig. 3a, b). Rigidity of overall atomic packing of the enzyme structure is conceivably the factor that determines stability of thermophilic GsFPPase and
$T t$ GGPPase. In GsFPPase, the residues are equivalent to Val17 and Cys73, therefore, Val17 and Cys73 are the residues that affect the thermal stability of GsFPPase. In fact, substitution of Cys 73 with Phe caused the enzyme to become sensitive to heat treatment (Koyama et al. 1994).

We also compared GsFPPase with FPPase from mesophilic bacteria Staphylococcus aureus (SaFPPase). Although $\mathrm{SaFPPase}$ shows a high degree of sequence identity (46\%) to GsFPPase, the two enzymes are from two different bacteria with distinct thermal properties (Donk

Table 1 Data collection statistics

\begin{tabular}{ll}
\hline Data collection & $\begin{array}{l}\text { Geobacillus stearothermophilus } \\
\text { FPPase }\end{array}$ \\
\hline Wavelength $(\AA)$ & 1.000 \\
Resolution range $(\AA)$ & $20.00-2.31$ \\
Space group & $\mathrm{P} 2{ }_{1} 2_{1} 2_{1}$ \\
Unit cell & $a=49.02 \AA, b=95.16 \AA$, \\
& $c=103.35 \AA$ \\
& $\alpha=90.00^{\circ}, \beta=90.00^{\circ}$, \\
No. of unique reflections & 20,709 \\
Completeness $(\%)$ & 99.34 \\
Average $I / \sigma(I)$ & 19.3 \\
$R_{\text {work }} / R_{\text {free }}$ & $0.211 / 0.252$ \\
RMS bond lengths $(\AA)$ & 0.011 \\
RMS bond angles $\left({ }^{\circ}\right)$ & 1.432 \\
Mean B value $\left(\AA^{2}\right)$ & 49.194 \\
No. of atoms & 4029 \\
Water molecules & 32 \\
Ramachandran favored $(\%)$ & 95 \\
Ramachandran allowed $(\%)$ & 3 \\
Ramachandran outliers $(\%)$ & 2 \\
\hline
\end{tabular}


Table 2 Computed phase transition temperature $\left(T_{\mathrm{p}}\right)$ values versus experimental melting point temperature $\left(T_{\mathrm{m}}\right)$ values

\begin{tabular}{lll}
\hline Enzyme & $\begin{array}{l}\text { Phase transition } \\
\text { point } T_{\mathrm{p}}\left({ }^{\circ} \mathrm{C}\right)\end{array}$ & $\begin{array}{l}\text { Melting point } \\
T_{\mathrm{m}}\left({ }^{\circ} \mathrm{C}\right)\end{array}$ \\
\hline Wild-type GsFPPase & 69.6 & 72 \\
C73F & 53.7 & $55^{\mathrm{a}}$ \\
Human FPPase & 44.7 & $50^{\mathrm{b}}$
\end{tabular}

${ }^{\text {a }} \mathrm{C} 73 \mathrm{~F}$ lost its enzymatic activity after heat treatment at $55^{\circ} \mathrm{C}$ for 30 min (Koyama et al. 1994)

b $T_{\mathrm{m}}$ value was taken from reference (Liu et al. 2014)

1920; Hughes and Hurst 1980). The remarkable difference between these two enzymes is the size of amino acid residue located on the 13th position before FARM (Fig. 4). Ile69 of $S a$ FPPase is more bulky than Cys73 of GsFPPase, which indicates that size of the residue in this position is one of the possible structural features contributing to thermostability of FPPases.

\section{Relation of Cys73 and the rigidity of GsFPPase structure}

We used Constraint Network Analysis (CNA) to investigate the relationship between Cys73 and the rigidity of GsFPPase structure. The dataset used in this study contains human FPPase (PDB ID: 4XQT), wild-type GsFPPase (PDB ID: 5AYP) and point-mutated GsFPPase (C73F) structures, with all of the structures in the open form (in the absence of a ligand). The calculated $T_{\mathrm{p}}$ and experimental $T_{\mathrm{m}}$ of enzymes from the dataset are given in Table 2. Unfortunately, $T_{\mathrm{m}}$ is not available for $\mathrm{C} 73 \mathrm{~F}$, so we used the temperature in which $\mathrm{C} 73 \mathrm{~F}$ lost most of its enzymatic activity. From Table 2, we see that the calculated $T_{\mathrm{p}}$ is in good agreement with the experiment. Both of the results showed that the thermostability of $\mathrm{C} 73 \mathrm{~F}$ is lower than the wild-type of GsFPPase, but higher when compared with human FPPase. To characterize the microscopic factors of these features, we further analyzed the stability map of
Fig. 5 Stability maps of enzymes from the dataset. Relative strengths of rigidity contacts are color-coded: pairs of residues where no or only a weak rigid contact exists are shown in red, and pairs of residues with strong rigid contacts are shown in blue. Rigid contacts of semiconserved regions are indicated with black dashed lines. Rigid contacts between $\alpha 1$ and $\alpha 10$ are indicated with green dashed lines. a Stability map of wildtype $G s$ FPPase. b Stability map of point-mutated GsFPPase C73F. c Stability map of human FPPase (a)

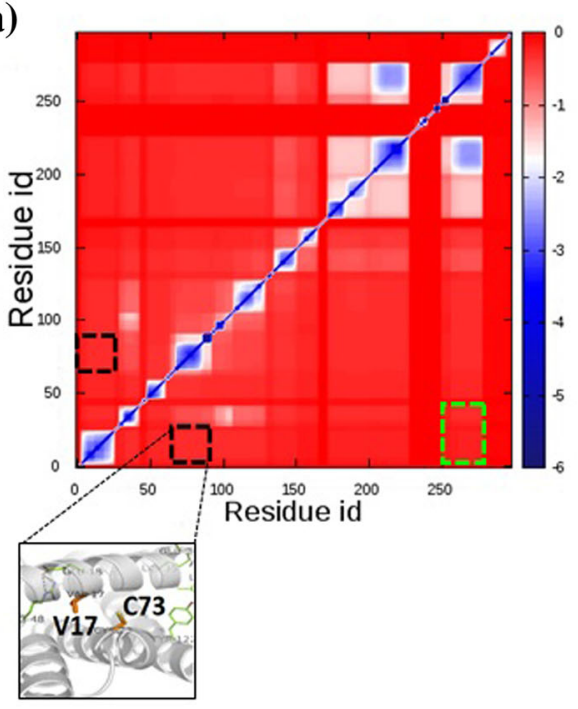

(b)

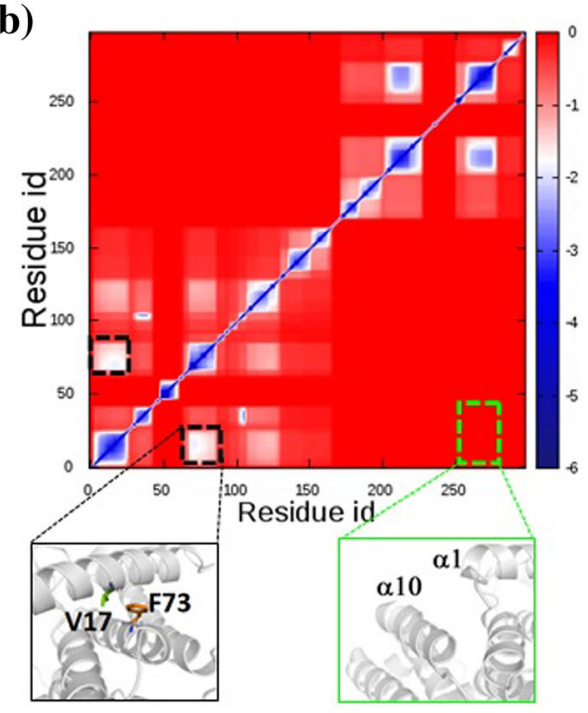

(c)

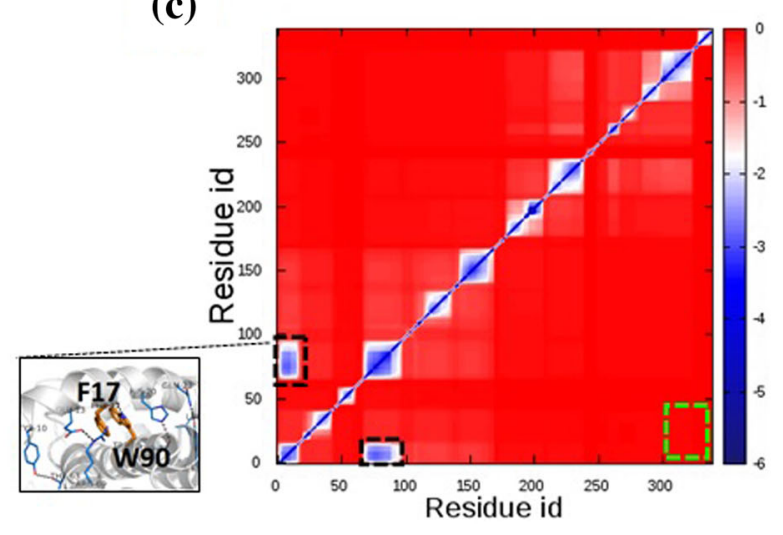


each enzyme. The stability map indicated rigid contacts between two residues that belong to the same rigid cluster. Examining the stability maps (Fig. 5) reveals that the pattern of rigidity contacts of $\mathrm{C} 73 \mathrm{~F}$ becomes more similar to that of human FPPase, although C73F still maintains the basic pattern of rigidity contacts of GsFPPase. When Cys 73 is replaced with Phe, the strength of rigidity contacts between amino acid 73 with Val17 and with its surrounding residues increases (indicated with black-dashed lines in Fig. 5b). On the other hand, strength of rigidity contacts of remaining residues in $\mathrm{C} 73 \mathrm{~F}$ decreases, which also means that the thermostability of C73F decreases, compared with wild-type GsFPPase. These simulation results support our hypothesis that replacement of Cys73 with a more bulky amino acid tightens the atomic packing of helices $\alpha 1$ and $\alpha 4$, which then loosens the atomic packing of the remaining helices (e.g. atomic packing between $\alpha 1$ and $\alpha 10$ ).

To the best of our knowledge, this is the first work that shows that prenyltransferases have "semi-conserved" regions that are crucial for structural stability, and shows how these regions affect the thermostability of the enzyme. Knowledge about thermostability factors of FPPase will open the way for protein engineering to increase its thermostability, and thus widen the industrial applications of FPPase in the future.

Acknowledgments We thank Mr. Daisuke Takeda for his technical assistance. We thank the BL-5A beamline staff at the KEK Photon Factory (Tsukuba, Japan) for their technical support. This work (partly) was supported by the Sasakawa Scientific Research Grant from The Japan Science Society (Grant No. 28-328).

\section{Compliance with ethical standards}

Conflict of interest All authors declare that they have no conflicts of interest to this work.

Open Access This article is distributed under the terms of the Creative Commons Attribution 4.0 International License (http:// creativecommons.org/licenses/by/4.0/), which permits unrestricted use, distribution, and reproduction in any medium, provided you give appropriate credit to the original author(s) and the source, provide a link to the Creative Commons license, and indicate if changes were made.

\section{References}

Chen A, Poulter CD (1993) Purification and characterization of farnesyl/geranylgeranyl diphosphate synthase. J Biol Chem 268:11002-11007

Chen A, Kroon PA, Poulter CD (1994) Isoprenyl diphosphate synthases: protein sequence comparisons, a phylogenetic tree, and predictions of secondary structure. Protein Sci 3:600-607

Donk PJ (1920) A highly resistant thermophilic organism. J Bacteriol 5:373-374

Eberhardt NL, Rilling HC (1975) Prenyltransferase from Saccharomyces cerevisiae. J Biol Chem 250:863-866
Fiser A, Do RKG, Sali A (2000) Modeling of loops in protein structures. Protein Sci 9:1753-1773

Hughes A, Hurst A (1980) The effect of $\mathrm{NaCl}$ on the upper temperature limit for growth of and enterotoxin synthesis by Staphylococcus aureus. Can J Microbiol 26:507-510

Kobayashi M, Koyama T, Ogura K, Seto S, Ritter FJ, BrüggemannRotgans IEM (1980) Bioorganic synthesis and absolute configuration of faranal. J Am Chem Soc 102:6602-6604

Koyama T, Saito A, Ogura K, Seto S (1980) Substrate specificity of farnesylpyrophosphate synthetase. Application to asymmetric synthesis. J Am Chem Soc 102:3614-3618

Koyama T, Ogura K, Baker FC, Jamieson GC, Schooley DA (1987) Synthesis and absolute configuration of 4-methyl juvenile hormone I (4-MeJH I) by a biogenetic approach: a combination of enzymatic synthesis and biotransformation. J Am Chem Soc 109:2853-2854

Koyama T, Obata S, Osabe M, Takeshita A, Yokoyama K, Uchida M, Nishino T, Ogura K (1993) Thermostable farnesyl diphosphate synthase of Bacillus stearothermophilus: molecular cloning, sequence determination, overproduction, and purification. J Biochem 113:355-363

Koyama T, Obata S, Saito K, Takeshita-Koike A, Ogura K (1994) Structural and functional roles of the cysteine residues of Bacillus stearothermophilus farnesyl diphosphate synthase. Biochemistry 33:12644-12648

Koyama T, Gotoh Y, Nishino T (2000) Intersubunit location of the active site of farnesyl diphosphate synthase: reconstruction of active enzymes by hybrid-type heteromeric dimers of sitedirected mutants. Biochemistry 39:463-469

Krüger DM, Rathi PC, Pfleger C, Gohlke H (2013) CNA web server: rigidity theory-based thermal unfolding simulations of proteins for linking structure, (thermo-) stability, and function. Nucleic Acid Res 41:W340-W348

Laskowski RA, Rullmannn JA, MacArthur MW, Kaptein R, Thornton JM (1996) AQUA and PROCHECK-NMR: programs for checking the quality of protein structures solved by NMR. J Biomol NMR 8:477-486

Liu YL, Lindert S, Zhu W, Wang K, McCammon JA, Oldfield E (2014) Taxodione and arenarone inhibit farnesyl diphosphate synthase by binding to the isopentenyl diphosphate site. Proc Natl Acad Sci USA 111:E2530-E2539

Maki Y, Kurihara M, Endo T, Abiko M, Saito K, Watanabe G, Ogura K (1995) Farnesyl diphosphate synthase reactions with dimethylallyl diphosphate analogues having oxygen atoms in their chains. Chem Lett 24:389-390

Ogura K, Koyama T (1998) Enzymatic aspects of isoprenoid chain elongation. Chem Rev 98:1263-1276

Pettersen EF, Goddard TD, Huang CC, Couch GS, Greenblatt DM, Meng EC, Ferrin TE (2004) UCSF Chimera-A visualization system for exploratory research and analysis. J Comput Chem 25:1605-1612

Pfleger C, Rathi PC, Klein DL, Radestock S, Gohlke H (2013a) Constrain network analysis (CNA): a python software package for efficiently linking biomacromolecular structure, flexibility, (thermo-) stability, and function. $\mathrm{J}$ Chem Inf Model 53:1007-1015

Pfleger C, Radestock S, Schmidt E, Gohlke H (2013b) Global and local indices for characterizing biomolecular flexibility and rigidity. J Comput Chem 34:220-233

Poulter CD, Rilling HC (1978) The prenyl transfer reaction. Enzymatic and mechanistic studies of the $1^{\prime}-4$ coupling reaction in the terpene biosynthetic pathway. Acc Chem Res 11:307-313

Radestock S, Gohlke H (2008) Exploiting the link between protein rigidity and thermostability for data-driven protein engineering. Eng Life Sci 8:507-522 
Radestock S, Gohlke H (2011) Protein rigidity and thermophilic adaptation. Proteins 79:1089-1108

Robert X, Gouet P (2014) Deciphering key features in protein structures with the new ENDscript server. Nucl Acids Res 42:W320-W324
Schellman JA (1997) Temperature, stability, and the hydrophobic interaction. Biophys J 73:2960-2964

Wiederstein M, Sippl MJ (2007) ProSA-web: interactive web service for the recognition of errors in three-dimensional structures of proteins. Nucleic Acid Res 35:W407-W410 